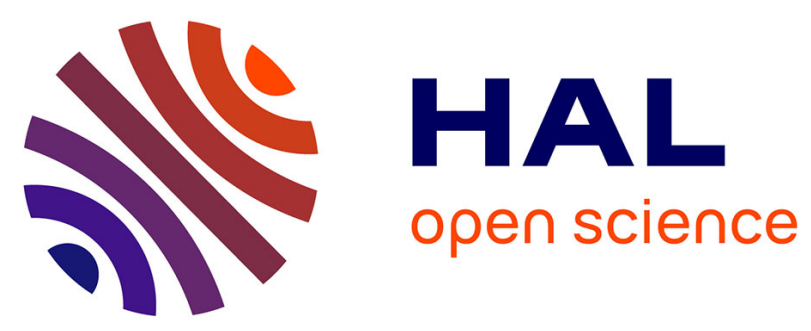

\title{
La percée originelle: entre aménagement urbain, transformation sociale et rentabilité financière
}

Pierre Fournier, Sylvie Mazzella

\section{To cite this version:}

Pierre Fournier, Sylvie Mazzella. La percée originelle: entre aménagement urbain, transformation sociale et rentabilité financière. Pierre Fournier, Sylvie Mazzella. Marseille entre ville et ports. Les destins de la rue de la république, La Découverte, pp.29-39, 2004. hal-01222029

\section{HAL Id: hal-01222029 \\ https://hal-amu.archives-ouvertes.fr/hal-01222029}

Submitted on 29 Oct 2015

HAL is a multi-disciplinary open access archive for the deposit and dissemination of scientific research documents, whether they are published or not. The documents may come from teaching and research institutions in France or abroad, or from public or private research centers.
L'archive ouverte pluridisciplinaire HAL, est destinée au dépôt et à la diffusion de documents scientifiques de niveau recherche, publiés ou non, émanant des établissements d'enseignement et de recherche français ou étrangers, des laboratoires publics ou privés. 
La percée originelle : entre aménagement urbain, transformation sociale et rentabilité financière

\author{
Pierre Fournier,', Sylvie Mazzella*
}

Au moment de sa percée sous le second Empire, la rue Impériale est déjà inscrite dans un projet d'aménagement de l'articulation entre ville et ports à Marseille, en lien avec les opérations de construction menées dans les quartiers de la Joliette, d'Arenc et du Lazaret par P. Talabot (docks) et par J. Mirès puis E. Pereire (immeubles résidentiels). Il s'agit d'orienter la ville vers les bassins portuaires de la Joliette, de « tirer » les beaux quartiers vers des espaces qui, en 1860, viennent d'être créés, gagnés sur la mer à l'occasion de la construction du port moderne. Il s'agit de construire un nouveau quartier résidentiel et économique, en lien aussi avec la gare de chemin de fer inaugurée en 1848.

L'opération s'inscrit dans un grand mouvement national de transformations qu'on situe dans la continuité de la politique du préfet Haussmann à Paris. On y retrouve les mêmes acteurs collectifs que ceux dont Maurice Halbwachs a décrit les interactions pour Paris dans son ouvrage sur les expropriations [Halbwachs, 1909]. Dans cette veine, les travaux de Marcel Roncayolo sur les mécanismes de la planification urbaine et de la spéculation [Roncayolo, 1996a] restent d'actualité pour donner une intelligibilité aux transformations à grande échelle de Marseille et pour lire le jeu des uns et des autres comme un système d'acteurs qui façonnent les grands espaces.

Pour Halbwachs, le planificateur-expropriateur (l'État, la municipalité) est incapable de prévoir, d'anticiper, d'imposer quoi que ce soit en matière de développement urbain. Il obéit seulement à la pression des demandes de la population; il est une courroie de transmission. Le pouvoir politique, royal, impérial ou républicain, n'a ainsi rien décidé ni empêché en matière d'extension de Paris. Les mesures prises par lui ne sont là que pour légitimer, officialiser des aménagements effectués en dehors des directives prévues. Le spéculateur, directeur de

\footnotetext{
- Sociologue, LAMES-universite de Provence.

". Sociologue, LAMES-CNRS.
} 
grande société foncière par exemple, n'est pas non plus l'acteur isolé qui, selon une idée reçue, crée de toutes pièces un nouveau quartier sans être assuré de son peuplement. Il est en revanche un acteur collectif particulièrement bien informé des préoccupations de la population, et possède la capacité d'en anticiper les besoins. Halbwachs précise : « Si, au-delà du désordre actuel, le spéculateur prévoit une organisation enfin stable, c'est qu'il prolonge et développe en pensée certains faits qui ont retenu son attention, c'est qu'il compare avec raison des cas dont lui seul reconnaît l' analogie, c'est que, par "une sympathie" particulière, il comprend mieux un certain nombre de phénomènes et en calcule plus exactement l'importance et la durée, c'est surṭut qu'il embrasse d'un seul regard une quantité de faits complexes et éloignés » [Halbwachs, 1909, p. 369].

À Marseille, les spéculateurs Jules Mirès, Paulin Talabot ou Émile Pereire correspondent à ce type d'acteur collectif, engagé dans de grands ouvrages haussmanniens caractérisés par le recours à la nouvelle procédure de déclaration d'utilité publique qui autorise les expropriations via la municipalité, et par l'intervention financière de grandes sociétés immobilières et de banques. Les raisonnements de M. Halbwachs, déplacés dans le contexte marseillais, permettent d'éclairer le sens et la portée de l'action menée par ces investisseurs, en même temps, peut-être, que les conditions de l'échec financier de l'opération de la rue Impériale.

\section{LES TRANSFORMATIONS URBAINES ET LEURS ACTEURS}

La construction rapide de la rue Impériale entre 1862 et 1867 s'inscrit dans un contexte de plein essor urbanistique dans lequel Marseille donne l'impression d'exploser, d'éclater en tous sens, et surtout vers l'est et le sud [Bailleux de Marisy, 1866, p. 621]. La population de la ville passe de 183000 habitants en 1846 à 300000 en 1866. Marseille connaît, durant cette période, une profonde mutation économique affectant jusqu'à la topologie de la ville : arrivée de la première ligne de chemin de fer, entrée des eaux de la Durance, avec l'implantation du nouveau port de la Joliette (1844-1854), construction massive d'immeubles et de bâtiments monumentaux pour les pouvoirs publics (le palais de justice 1856-1862, la préfecture 1862-1866), création de parcs comme celui qui jouxte le palais Longchamp (1862-1864), exécution de travaux de voirie qui redéfinissent l'extension de la ville. Différents facteurs locaux et nationaux fav̌orisent l'ensemble de ces réalisations dans Marseille, notamment le souhait des maires (Honnorat, Lagarde, Onfroy, Rouvière, Bernex...) et préfet (de Maupas) de mener des politiques monumentales, couplé à la transformation des conditions de financement de ce type d'opérations avec la fondation d'une nouvelle forme de banque, qui recueille les capitaux du grand public et les transforme en pî̀ts à long terme (le Crédit mobilier fondé en 1852 par les frères Pereire, la Caisse nationale des chemins de fer fondée en 1853 par J. Mirès...). 
Le percement de la rue Impériale répond non seulement à la nécessité d'accompagner le développement urbain en coordonnant ces transformations localisées, mais aussi au problème des vieux quartiers insalubres du centre historique et populaire (photo 7, la rue de Lorette avant sa destruction) qui occupe les débats publics depuis le $\mathrm{XVm}^{e}$ siècle [Roncayolo, 1996a, p. 456]. Après l'extension de la ville au sud aux XVIr et XVII' siècles, on parle d'une troisième ville en train de se construire, au nord cette fois, sur les terrains en arrière des quais du nouveau port. Les écarts dans la taille du parcellaire sont frappants, avec des parcelles de petite taille, distribuées suivant des rues peu rectilignes pour la ville médiévale au nord du port naturel, avec des îlots géométriques de taille plus importante pour la ville moderne au sud, avec enfin de gros îlots très symétriques en arrière des nouveaux bassins portuaires ( $c f$. carte). Avant l'opération de la rue Impériale qui débute en 1862, c'est la construction de ces nouveaux quartiers sur les terrains de la Joliette, soutenue par le spéculateur Jules Mirès dès 1856, que Marcel Roncayolo situe au « démarrage » de l'haussmannisation marseillaise par le type de bâti standardisé qu'on y rencontre et par l'effet d'entrainement qu'elle exerce durant les années 1860 [Roncayolo, 1996a, p. 223]. Mirès achète au prix de 50 francs le $\mathrm{m}^{2}$ un terrain de $391522 \mathrm{~m}^{2}$ gagnés sur la mer à mesure de la construction du nouveau port qui se fait par remblai jusqu'à atteindre la cote requise par le tirant d'eau des bateaux attendus. Il parie sur une importante rente spéculative ${ }^{1}$ et laisse écrire, dans le Journal des chemins de fer dont il est le directeur, que ces terrains ont une valeur foncière comprise entre 300 et 400 francs le $\mathrm{m}^{2}$. Pour relier cette troisième ville aux deux précédentes de part et d'autre du port naturel, le passage par les quais apparaît comme un long détour, d'autant qu'ils sont souvent encombrés par des marchandises. Quant au prolongement vers le nord de l'axe de la rue d' Aix, qui structure le lien du certre ancien avec la ville du sud via le cours Belsunce et la rue de Rome, il présente l'inconvénient de dénivelés considérables ralentissant la circulation. Plusieurs solutions sont envisagées qui vont du tunnel sous la colline (projet Vaucher en 1855) à l'arasement complet de cette barrière physique (projet Mirès en 1858). Le percement d'une large rue dans le bâti ancien de la ville originelle et dans l'épaisseur de la colline (photo 8) se situe à l'entre-deux. Par ses caractéristiques, l'ouverture de la rue Impériale constitue déjà une mutation urbaine considérable dont la réalisation apparaît comme démesurée aux contemporains [Fabre, 1869, p. 367-368]. Une tranchée d'une profondeur de 25 mètres par endroits, d'une largeur de 60 mètres, est creusée; $1200000 \mathrm{~m}^{3}$ de déblais sont déversés dans la mer grâce à quatre lignes de chernin de fer établies sur 12 kilomètres (photo 9); pour la première fois en France, on utilise, sur un chantier de construction, des locomotives, des grues roulantes de 35 mètres de hauteur (photo 10) et des machines à fabriquer le mortier.

1. J. Lautman note que les terrains vierges sont toujours très appréciés des promoteurs pour la forte plus-value qui peut y être réalisée, sachant que, " bon gré mal gré, collectivités locales et puissance publique seront bien obligées de réaliser les travaux d'adduction, de voirie, de construction d'écoles et de creation de lignes de transport " [Lautman, 1969, p. 623]. 


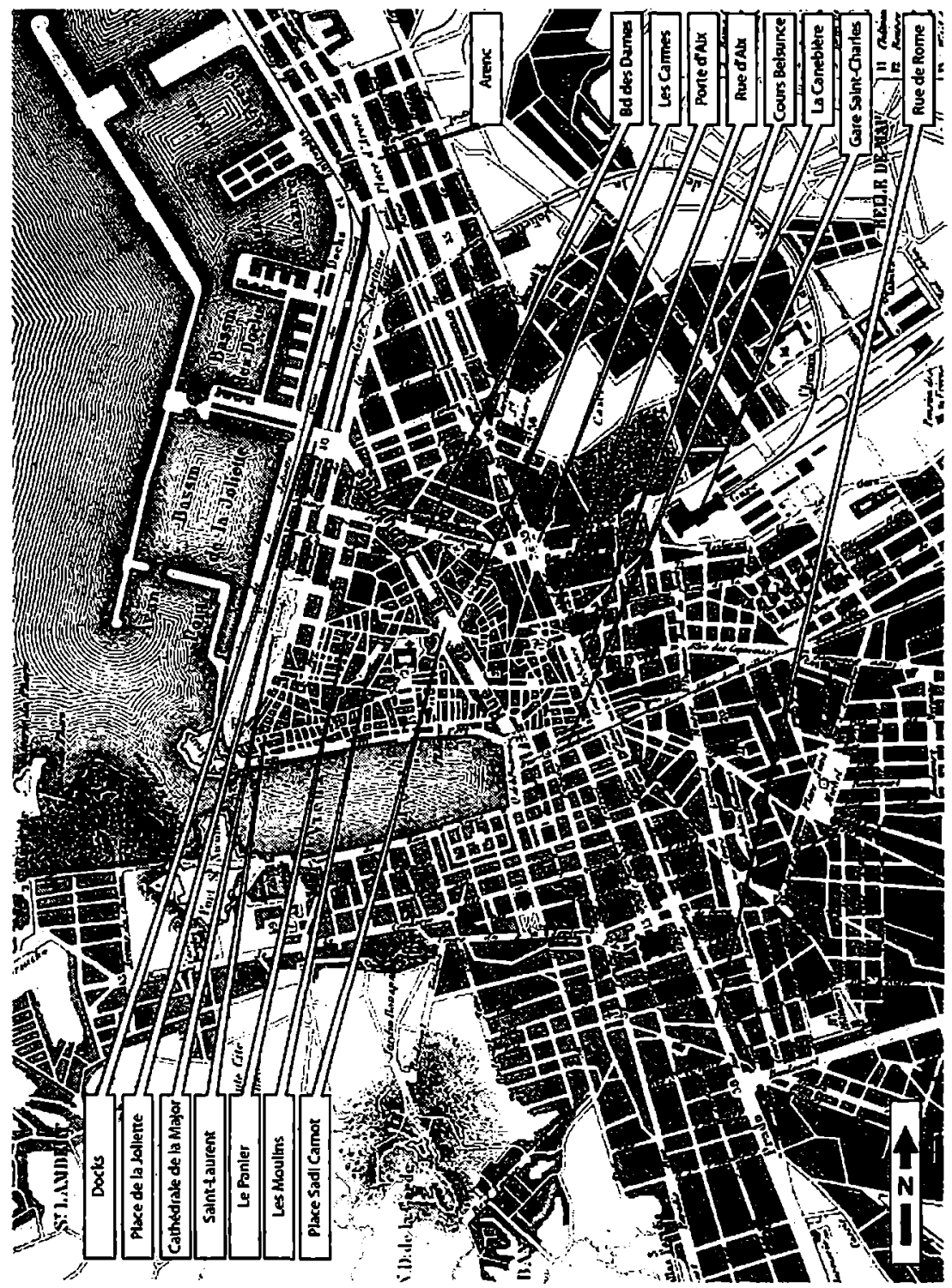

Source : Arlas national. Paris, Fayard, 1874. Mise en forme : Thomas Bresson. 
Un soir, à dix heures, par une lune blanche et un ciel immaculé, j'ai suivi la nouvelle rue qui joint les deux ports. On a enlevé une colline pour la frayer. La ville a vendu le terrain vingt millions; les frères Pereire y bâtissent pour trente à quarante millions de maisons, toutes les maisons à la fois, chacune d'elles monumentale et monstrueuse, haute de six étages et, si je calcule d'après mon bôtel, avec des escaliers de cent cinquante marches. Toutes sont en larges pierres de taille, blanches, sculptées, les unes achevées, d'autres à demi finies, d'autres sortant de terre parmi les échafaudages, les grues, les treuils à vapeur, les larges échappées d'eau coulante. La rue ressemble à quelque Baalbeck non achevée et déserte. En effet, sous l'Empire romain, on bâtissait des cités d'un coup comme aujourd'hui, par accumulation de capitaux et concentration administrative.

TalNe H. (1897), Carnets de voyage. Notes sur la province (1863-1865), Paris, Hachette, p. 314-315.

La procédure d'expropriation est rapide au regard de l'importance de la surface ( $100000 \mathrm{~m}^{2}$ pour la nouvelle voirie et pour les futurs immeubles) : six mois pour les ententes amiables entre juillet 1861 et janvier 1862, huit mois pour la majeure partie des règlements par jury d'expropriation. 935 maisons sont détruites, 16000 personnes déplacées vers de nouveaux immeubles, construits par les spéculateurs dans les quartiers de la Belle-de-Mai et de Notre-Dame-de-la-Garde [Jasmin et Jasmin, 1994, p. 14]. Il faut, en effet, se garder d'imaginer des spéculateurs intéressés seulement par la construction d'immeubles bourgeois à cette époque. C'est ce que montre M. Lescure à propos de la dynamique à l'auvre pour le quartier populaire de Clignancourt dans le XVIII ${ }^{c}$ arrondissement de Paris en 1882. La rente foncière y découle de l'écart entre faibles coûts d'acquisition et de construction et forts volumes vendus du fait de la densification du bâtî standardisé par rapport au bâti populaire antérieur, avec pour effet une «transformation du quartier par un double processus d'élimination des populations les plus défavorisées et de promotion verticale de ses occupants. La structure des loyers [... ], les façades en pierre de taille, les vestibules en stuc, le confort donné aux appartements (eau, gaz, sonnettes) en réservent l'accès à une élite ouvrière, stabilisée géographiquement et avide d'intégration sociale » [Lescure, 1980, p. 45]. Ainsi, à Marseille, quand Mirès pense à englober la vieille ville dans son projet et propose, en 1858, une opération d'unification des nouveaux quartiers de la Joliette à l'ancien port, qui suppose, on l'a vu, de raser les trois collines de la vieille ville (Saint-Laurent, les Moulins, les Carmes) qui dominent le Vieux-Port et l'abritent du vent, il s'agit d'augmenter encore les perspectives de rente associées à ce type d'opérations d'aménagement dans la troisième ville.

«La vieille ville de Marseille est siruée sur une éminence dont l'élévation sur certains points n'est pas moindre de 40 mètres. De cet amas de 3000 maisons, la plupart en ruine et faisant honte à la civilisation, s'exhalent les émanations les plus dangereuses pour la santé publique. Dans ce foyer d'infection où naquit 
la peste de 1720 , se presse une population agglomérée de 50000 âmes. La ville de Marseille proprement dite et l'ancien port, placé au pied de la vieille ville, sont séparés des nouveaux quartiers et des nouveaux ports par la vieille ville dont les rues sales et si étroites ne sont accessibles qu'aux piétons qui osent en affronter l'escalade ${ }^{2}$. "

À moins qu'il ne s'agisse, pour Mirès, de trouver in extremis une solution pour assurer le succès compromis des premières réalisations. On peut se poser la question au regard de la délibération du conseil municipal du 1 1er décembre 1860 qui précise que les dépenses de construction dans le quartier de la Joliette et dans celui d'Arenc ont été supérieures à celles qui étaient prévues et que les locations s'effectuent très difficilement. Les étages inférieurs des immeubles du quai de la Joliette restêtt inoccupés alors que Mirès avait espoir de voir s'y implanter les corps consulaires et les grandes compagnies de navigation [Leverne, 1925, p. 30].

Émile Pereire, président du conseil d'administration de la Compagnie immobilière, fonde, lui aussi, à cette époque, beaucoup d'espoirs sur Marseille, « dont la prospérité est née des causes générales qui existent partout où le progrès du crédit et l'établissement des chemins de fer ont imprimé une grande impulsion au commerce et à l'industrie. Elle est favorisée de plus par une position exceptionnelle et par le commerce avec notre grande colonie qui a pris des proportions inouies et devra s'accroître lorsque le percement du canal de Suez aura centralisé le commerce des Indes dans le bassin de la Méditerrané ${ }^{3}$ ". Mais, à cette époque, contrairement à Mirès et à Talabot, il n'a pas encore véritablement sa place parmi les investisseurs en lien avec le nouveau port alors qu'il souhaiterait y faire arriver un prolongement de la ligne Bordeaux-Sète qu'il a construite lorsqu'il était à la tête de la Compagnie du Midi [Richard, 1998, p. 173]. À la faillite de J. Mirès, E. Pereire s'intéresse donc au projet de la rue Impériale encore à construire et intègre l'actif de la Société des ports dans sa Compagnie immobilière ${ }^{4}$. Le 14 août 1862, il signe avec le maire, Balthazar Rouvière, un traité par lequel la Ville vend à la Compagnie immobilière tous les terrains en bordure de la rue Impériale et des rues nouvelles adjacentes, d'une superficie minimale de $60000 \mathrm{~m}^{2}$ au sol'. Le prix de vente est fixé à 300 francs le $\mathrm{m}^{2}$, soit un total de 18 millions de francs.

2. Propos de Mirès dans sa lettre au procureur général près de la Cour de cassation Dupin, À mes juges, ma vie, et mes affaires, Paris, Imprimerie Vallée, 1862, 47 p. [Leverne, 1925, p. 45].

3. Assemblée générale de la Compagnie immobilière de Paris, 19 novembre 1862 [Leverne, 1925. p. 48-49].

4. En 1863, le patrimoine foncier de la Compagnie immobilière se compose à Marseille de l'exdomaine de la Société des ports, sur lequel 45 maisons sont bâties et 33 en cours d'achèvement, de la rue Impériale et de vingt rues attenantes, des terrains du boulevard des Darnes, des terrains des Catalans en lien avec des projets d'établissement de ports au sud. À ce moment-là en effet, il ne semble pas possible que les ports nord puissent être développés vers l'Estaque à cause de l'installation des bassins de radoub qui en ferment l'extrémité nord [Leverne, 1925, p. 50]. ;

5. En fait, elle passe à $70000 \mathrm{~m}^{2}$ après rétrécissement des largeurs de voie envisagées et surtout du diamètre de la place centrale, par souci d'équilibrer le budget de l'opération. 
La rue Impériale donne donc l'exemple d'une opération haussmannienne menée par différents acteurs collectifs : les planificateurs-expropriateurs (l'État et la municipalité) et les spéculateurs, avec, autour d'eux, la myriade d'actionnaires regroupés dans des compagnies immobilières soutenues par le nouveau système bancaire que ces sociétés ont fondé, mais aussi les architectes, les petits entrepreneurs bâtisseurs d'immeubles, c'est-à-dire de segments de la rue suivant un cahier des charges fixé par la Compagnie immobilière, et les ingénieurs des Ponts et Chaussées. Auguste Gassend, ancien chef de bureau de la direction des Ponts du département, est de ceux-ci. Il a alors la responsabilité de la voirie et des travaux publics à la mairie de Marseille. C'est lui qui, avec le maire Louis Lagarde, convainc Napoléon III, en septembre 1860, d'approuver le projet d'ouverture d'une route nationale empruntant ce tracé - ici $n^{\circ} 8$ bis, allant de Marseille vers l'Italie -, avec pour enjeu le financement par l'État de 30\% des travaux [Darin, 1988, p. 482]. C'est encore lui qui précise, plus tard, le tracé du plan, le parcellaire, les îlots à démolir, en concertation avec les membres de commissions spécialement constituées pour examiner et approuver les pians proposés [Gassend, 1867, p. 14-176]. Deux commissions spéciales d'examens des plans illustrent cette variété d'acteurs. En 1860, sous l'égide du préfet, le sénateur de Maupas, la première réunit le président de la chambre de commerce, le secrétaire général de l'évêché, un membre du Conseil général, le receveur général, le président du Grand Conseil des sociétés de prévoyance et de secours mutuel. Une seconde commission, réunie en 1862, poursuit la négociation. Elle est constituée d' acteurs locaux : un avocat, le directeur de l'École de médecine, un banquier, un négociant, un membre de la chambre de commerce et un juge au tribunal de première instance. Elle a pour objectif, précise Gassend, de recueillir les observations du public pour qu'il puisse formuler son avis. S'il faut compter avec les résistances du pouvoir local, comme en témoigne la longue polémique relative aux projets d'extension portuaire tout au long du xxe siècle [Bonillo, 1992, chap. 3], cette lourde procédure de négociation ne doit pas masquer le poids décisif du corps des Ponts et Chaussées qui sélectionne les projets soumis aux enquêtes publiques et définit les choix techniques. Et si les tractations à l'échelle locale ont su ici s'articuler avec la volonté nationale pour ia réalisation du projet', c'est peutêtre du fait que certains acteurs ont occupé successivement des positions de représentants de l'intérêt de l'État et de représentants des intérêts locaux, comme de Montricher, ingénieur du Service maritime du département avant de démissionner pour devenir inspecteur général des Travaux publics de Marseille, ou Gassend qui, de chef de bureau à la direction des Ponts et Chaussées des Bouches-du-Rhône, passe à la direction des Travaux marseillais [Jasmin et Jasmin, 1994, p. 11].

6. Une Commission des travaux publics est instaurée pour traiter de ces questions. Elle réunit des membres civils et militaires. Elle est ensuite démultipliée en commissions ad hoc par chantiers.

7. $D^{\prime}$ autres projets de rénovation des quartiers de la vieille ville au début du $x x^{e}$ siècle, portés par les urbanistes Jacques Greber et Eugène Beaudouin, n' aboutissent pas, en partie faute d'avoir su articuler ces deux niveaux de décision. 


\section{L'ÉCHEC D'UNE OPÉRATION FINANCIÈRE}

Tout un jeu de crédits, d'avances, d'intérêts mais aussi d'endettements en cascade s'installe entre les différents acteurs du projet [Dubois, 1978-1980, p. 50-52]. En 1860, l'État accorde une subvention de 6 millions de francs à la municipalité pour engager les travaux dans la rue Impériale $\mathrm{e}^{8} \mathrm{et}$ l' autorise à emprunter pour poursuivre les travaux d'aménagement des nouveaux ports. La Ville, qui a programmé une subvention de 12 millions de francs de plus pour équilibrer le budget, autorise un crédit à la Compagnie immobilière d'Émile Pereire. La même Compagnie immobilière consét des emprunts hypothécaires aux petits entrepreneurs qui lui achètent des parcelles de la rue ${ }^{9}$ pour y construire des immeubles et en revendre les appartements ${ }^{10}$. En cas d'échec, les difficultés s'enchaînent dans l'ordre inverse. Lorsque les petits entrepreneurs ne parviennent pas à vendre ni à louer ces appartements, leur faillite fait du bailleur de fonds le propriétaire. Mais la Compagnie immobilière n'arrive pas davantage à vendre ni à louer, donc à rembourser ses dettes, et est mise en liquidation à son tour en 1872. La municipalité augmente par suite son endettement auprès de l'État. Au lieu des 12 millions de francs qu'elle croyait engager dans l'opération, c'est 30 millions que Marseille y engloutit [Jasmin et Jasmin, 1994, p. 15].

La Société immobilière marseillaise est constituée en 1878 à la demande de la Société marseillaise de crédit et des grands noms du patronat négociant marseillais qui en sont les principaux actionnaires (comme Amédée Armand, Théophile Roccoffort, Émile Darier, Étienne Zafiropulo, Henry Bergasse, Emest Rondel, Alphonse Grandval), pour éviter que leurs placements dans le programme MirèsPereire perdent toute valeur dans la liquidation. Elle reprend d'abord de la Compagnie immobilière l'ancien actif immobilier de la Société des ports (comprenant les immeubles et terrains des nouveaux quartiers de la Joliette et du Lazaret) [Leverne, 1925 , p. 84]. Vient s'ajouter, en 1890 , le reste des actifs de la Compagnie immo-

8. Dans le budget prévisionnel de l'opération, cela représente un tiers des $18 \mathrm{MF}$ à couvrir, à côté des $18 \mathrm{MF}$ reçus de la vente des terrains à J. Mirès, pour équilibrer les frais de $30 \mathrm{MF}$ d'expropriation et les $6 \mathrm{MF}$ de frais de voirie.

9. Cela conceme 10 parcelles sur 18 [Jasmin et Jasmin, 1994, p. 15]. Certe pratique qui conduit à ce que « la Compagnie immobilière [prenne] place parmi les organismes de crédit immobilier » est précisément décrite par M. Lescure [1980, p. 27-28]. Le rôle des entrepreneurs de bâtiments est, dès lors, réduit à celui d'agents d'exécution, seules les grandes sociétés immobilières ayant à charge la responsabilité financière et commerciale de l'opération par la masse de leurs capitaux et par leurs relations avec le pouvoir [ibid., p. 65].

10. Si, à partir du second Empire, un type nouveau de propriélaire s' affirme, souvent homme d'affaires, noble parfois, le propriétaire d'immeuble n'est pas toujours en possession d'un patrimoine important. "Artisans et boutiquiers s'y risquent en recourant à l'emprunt hypothécaire : l'opération n'est couronnée de succès que si, les affaires marchant par ailleurs, les loyers garantissent plus que le remboursement » [Magni, 1996, p. 407]. 
bilière (comprenant les immeubles de la nue de la République et les terrains d'Arenc) en provenance de la Nouvelle Compagnie immobilière qui était née, en 1881, de la liquidation définitive de la Compagnie immobilière. Tout cela se fait dans un environnement plus favorable : la rue de l'Impératrice, imaginée dès l'origine comme susceptible de valoriser le site ${ }^{11}$, renommée rue Colbert, et espérée pour 1883, se réalise enfin, tout comme la liaison ferroviaire entre l'Estaque et la Joliette, et la construction de la gare d'Arenc.

Comment expliquer l'échec financier de l'ouverture de la rue dans les années 1860 ? En 1881, les liquidateurs de la Compagnie immobilière analysent la situation avec recul : «Plusieurs causes ont longtemps retardé le développement de nos locations à Marseille : un ralentissement marqué dans l'accroissement de la population, l'inexécution des grands travaux d'utilité publique, les habitudes et les traditions qui tenaient les locataires, industriels et commerçants éloignés des nouveaux quartiers, enfin le trop grand nombre d'immeubles bâtis eu égard au chiffre de la population ${ }^{12}$. » Pour les seules années 1861 à 1863, 2640 logements ont été construits alors que la moyenne annuelle de construction à Marseille était de 350 logements entre 1857 et 1861 [Leverne, 1925, p. 62]. La Compagnie immobilière est ainsi contrainte de renoncer à vendre ses logements ${ }^{13}$; elle ne parvient à louer la totalité du parc de la rue de la République qu'à partir de 1880.

L'échec financier de la rue Impériale est un exemple de discordance entre les attentes des spéculateurs et le comportement des habitants : alors que les prix des terrains en 1860 sont parmi les plus élevés de Marseille ${ }^{14}$, la rue reste en partie inhabitée jusqu'en 1880. La population n'y vient pas, poursuivant son lent déplacement vers le sud de la ville, commencé dès le xvتr siècle, pour sa fraction bourgeoise; s'installant sur les terrains laissés vierges et en périphérie, suivant une logique libérale d'urbanisation, pour sa fraction populaire [Roncayolo, 1996a, p. 464]. Cela signifie l'échec de la " troisième ville ». Comment le comprendre? C'est pourtant dans cette zone de la ville qu'il y a le plus nettement anticipation de besoins collectifs. Et le développement de l'activité portuaire témoigne de sa pertinence. Dans ce cadre, on l'a dit, la nue Impériale doit servir à relier les « villes ».

11. Dans le projet Gassend-Delestrac de 1858 , sont prévues ( trois grandes voies reliant $1^{\circ}$ ) les ports; $2^{\circ}$ ) la place de l'Arc-de-Triomphe et par suite la gare de chernin de fer avec $l^{\prime}$ hôtel de ville; $3^{\circ}$ ) l'extrémilé du Cours [Belsunce] vers la rue d'Aix, avec la cathédrale [de la Major] en construction » [Gassend, 1867, p. 7]. C'est leur intersection qui décide de la position de la place Centrale, devenue place Sadi-Carnot, et de sa forme à six branches. Une réalisation en plusieurs temps est d'emblée envisagée, avec le concours de financements publics pour la première voie et s'appuyant sur le seul financement privé pour les autres.

12. Liquidation de la Compagnie immobilière. Rapport présenté à l'assemblée des actionnaires du 13 avril 1881, cité par Claude et Denise Jasmin [1994, p. 22, note 59].

13. Sur l'échec d'une tentative de vente en lots à l'Imperial Land City of Marseilles Lid, cf. Leverne [1925, p. 54].

14. Is sont nettement plus élevés que ceux qui ont été accordés à Mirès pour les terrains de la Joliette mais moins que le prolongement de la Canebière au même moment [Jasmin et Jasmin. 1994, note 50]. 
Il semble cependant que la compétition à laquelle se livrent les investisseurs privés (comme Mirès, Talabot, Pereire...) dans la nouvelle zone les fait jouer d'abord les uns contre les autres, et ici contre l'éventualité de cette troisième ville dont les bénéfices escomptés seraient jugés mal partagés. Il faudrait aussi évoquer le climat de concurrence qui prévaut, parallèlement, entre les acteurs publics en charge de l'aménagement, par exemple entre Gassend et Delestrac pour succéder à de Montricher [Jasmin et Jasmin, 1994, p. 11]. Bref, c'est une analyse en termes de champs du pouvoir (de champ des spéculateurs, de champ des planificateurs, peutêtre même de champ politique) que propose implicitement l'article de Claude et Denise Jasmin [1994]. La conséquence de ces tensions pour la rue Impériale est qu'il ne s'agit rapidement plus, avec ces projets, de relier une troisième ville aux précédentes, mais de rattacher de nouveaux quartiers au reste de la ville, de réunir la citér ${ }^{15}$. Cependant, Claude et Denise Jasmin en concluent sans doute trop vite que « le milieu qui venait d'être tranché et déplacé reprit et sa place et sa revanche " [Jasmin et Jasmin, 1994, p. 20] sans effet sur le peuplement urbain. Sur ce point d'un échec financier qui ne serait pas forcément synonyme d'absence de tout effet produit en termes de dynamique urbaine, il faut y regarder de plus près par une étude fine des transformations de la morphologie sociale qui fait l'objet de la deuxième partie de cet ouvrage. C'est sur cette question de l'anticipation de la dynamique urbaine qu'on doit réinterroger l'opération de la rue Impériale avec plus d'un siècle de recul, pour s'apercevoir que des besoins collectifs ont bien été satisfaits, mais entendus de façon particulière.

Pour l'heure, on voit là que, conformément à l'analyse d'Halbwachs, les besoins collectifs en termes de résidence ne se laissent pas guider par la seule volonté politico-économique; la ville ne se décrète pas par " accumulation de capitaux et concentration administrative ${ }^{16}$ ". Sur ce point d'un échec dans l'anticipation des besoins, l'analyse de $\mathrm{M}$. Halbwachs semble moins offrir un modèle prescriptif, auquel les spéculateurs marseillais ne se seraient pas conformés, qu'un outil pour interroger l'échelle des raisonnements des acteurs collectifs engagés dans ce genre d'opération. Ainsi, J. Mirès tente d'abord, avec le projet de la rue Impériale, une opération de sauvetage de son premier projet qui, lui, relevait bien d'anticipations sur les besoins collectifs en lien avec la croissance démographique de Marseille avant 1867. Quant à E. Pereire, il ne fait que suivre la commande municipale inspirée par Mirès, en reportant son rôle d'anticipation sur d'autres secteurs que ceux de la question résidentielle. Il juge pertinent le projet municipal sur la base d'éléments de contexte d'une autre nature, moins locaux, plus larges, moins résidentiels que commerciaux, situant la rue Impériale entre vieux et nouveau ports de commerce bien sûr, et entre la gare ferro-maritime qu'il projette à la Joliette

15. M. Roncayolo souligne la volonté de l'intervention publique et privée de « moins viser à ségréger qu'à unifier " [Roncayolo, 1996b, p. 57].

16. Formule des Camets de voyage de Taine. 
(qui ne voit le jour que vers 1890) et la gare PLM de Saint-Charles, à un moment où le développement économique semble passer par chacune de ces deux voies de communication sur lesquelles il parie à l'échelle nationale et internationale.

\section{BibLIOGRAPHIE}

BAIleUX De Marusy A. (1866), "La ville de Marseille, ses finances et ses travaux publics ", Revue des deux mondes, $1^{\text {et }}$ août 1866, p. 620-651.

BonLLo J.-L. [dir.] (1992), Marseille, ville et port, Marseille, Parenthèses, 221 p.

DARUN M. (1988), «Les grandes percées urbaines du Xx“ siècle : qualre villes de province », Annales $E S C, \mathrm{n}^{\circ} 2$, p. $477-505$.

Duвors H. (1978, 1979, 1980), « Une grande opération urbaine sous le second Empire. Le percement de la nue Impériale (rue de la République) ", Bulletin de la Société de géographie de Marseille, nouvelle série, $\square^{\circ} 13,1978-1979-1980$, p. 47-56.

Fabre A. (1869), Les Rues de Marseille, tome V, Marseille, Camoin, 505 p.

GASSEND A. (1867), La Rue Impériale de Marseille. Étude historique et archéologique, Marseille, Académie impériale des sciences, belles-lettres et arts, $35 \mathrm{p}$.

Halbwachs M. (1909), Les Expropriations et le prix des terrains à Paris (1860-1900), Paris, Comély, $415 \mathrm{p}$.

JASMN C., JASMIN D. (1994) « Marseille : la rue Impériale », Revue de l'art, $n^{\circ}$ 106, p. 11-22.

LAUTMAN J. (1969), «La spéculation, facteurd'ordre ou de désordre économique? » Revue française de sociologie, vol. X, p. 608-630.

Lescure M. (1980), Les Sociétés immobilières en France au XXX siècle. Contribution à l'histoire de la mise en valeur du sol urbain en économie capitaliste, Paris, Publications de la Sorbonne, $84 \mathrm{p}$.

LEVERNE P. (1925), Une grande entreprise immobilière, la Société immobilière marseillaise, Marseille, thèse de sciences politiques, Aix-en-Provence, faculté de droit et de sciences politiques, Marseille, Imprimerie Lecomte, $172 \mathrm{p}$.

MAGRI S. (1996), « Les propriétaires, les locataires, la loi. Jalons pour une analyse sociologique des rapports de location, Paris 1850-1920 \#, Revue française de sociologie, vol. 37, $n^{\circ} 3$, p. $397-418$.

RICHARD E. (1998), «Le percement de la rue Impériale », in Histoire de Marseille en treize événements, Marseille, Jeanne Laffitte, p. 163-177.

RonCAYOLo M. (1996a), Les Grammaires d'une ville. Essai sur la genèse des structures urbaines à Marseille, Paris, Éditions de l'École des hautes études en sciences sociales, $507 \mathrm{p}$.

- (1996b), Marseille. Les territoires du temps, Paris, Éditions locales de France, 135 p. 